\title{
Role of reflections in the generation of a time delay in strong-field ionization
}

\author{
Daniel Bakucz Canário $\odot,{ }^{*}$ Michael Klaiber $\odot$, Karen Z. Hatsagortsyan $\odot{ }^{\dagger}$ and Christoph H. Keitel $\odot$ \\ Max-Planck-Institut für Kernphysik, Saupfercheckweg 1, 69117 Heidelberg, Germany
}

(Received 20 January 2021; revised 31 May 2021; accepted 9 August 2021; published 7 September 2021)

\begin{abstract}
The problem of time delay in tunneling ionization is revisited. The origin of time delay at the tunnel exit is analyzed, underlining the two faces of the concept of the tunneling time delay: the time delay around the tunnel exit and the asymptotic time delay at a detector. We show that the former time delay, in the sense of a delay in the peak of the wave function, exists as a matter of principle and arises due to the sub-barrier interference of the reflected and transmitted components of the tunneling electronic wave packet. We exemplify this by describing the tunneling ionization of an electron bound by a short-range potential within the strong-field approximation in a "deep tunneling" regime. If sub-barrier reflections are extracted from this wave function, then the time delay of the peak is shown to vanish. Thus, we assert that the disturbance of the tunneling wave packet by the reflection from the surface of the barrier causes a time delay in the neighborhood of the tunnel exit.
\end{abstract}

DOI: 10.1103/PhysRevA.104.033103

\section{INTRODUCTION}

In both classical and quantum mechanics, the passage of time is always defined with respect to some dynamical variable (for instance, the hands of a clock). In the absence of a canonical quantum-mechanical time operator, the definition and measurement of time in quantum mechanics are challenging since dynamical observables are inherently nondeterministic. Despite the conceptual difficulties involved, operational techniques with which to measure time have been developed (see, e.g., Refs. [1-9] for overviews).

One of the main applications of these time measurement protocols is the study of the time taken for a particle to tunnel through a barrier, known as tunneling time. Several definitions of the tunneling time exist, such as the Wigner time [10-14], the Büttiker-Landauer time [15], the Pollak-Miller time [16], the Larmor time [17-21], the dwell time [22], etc. [23-27]. Each definition corresponds to a specific aspect of the measurement process and, in general, these do not coincide.

The tunneling phenomenon plays an essential role in the strong-field ionization process of atoms and in the related field of attoscience. State-of-the-art techniques in attosecond science are now able to provide exceptional time and space resolution, reaching tens of attoseconds time and Angström-space resolution. In particular, the attoclock technique provides time resolution of tunneling ionization [28], and has inspired researchers to experimentally address the

\footnotetext{
*daniel.bakucz-canario@mpi-hd.mpg.de

${ }^{\dagger}$ k.hatsagortsyan@mpi-hd.mpg.de
}

Published by the American Physical Society under the terms of the Creative Commons Attribution 4.0 International license. Further distribution of this work must maintain attribution to the author(s) and the published article's title, journal citation, and DOI. Open access publication funded by the Max Planck Society. challenging problem of tunneling time [29-33], i.e., how much time, if any, elapses during the quantum tunneling process.

This question strikes at the fundamentals of quantum mechanics but experiments have often been followed by controversial discussion [26,29-67] in the strong-field community arguing either for or against the existence of a tunneling induced time delay between ionized electron wave packet and ionizing laser field.

In this respect we emphasize the distinction between two concepts of the tunneling time in strong-field ionization, namely, the time delay near the tunnel exit (the classically expected coordinate for the tunneled electron to emerge) and the asymptotic time delay [37]. While the latter is relevant to attoclock experiments, the former, known also as the Wigner time delay, can be calculated theoretically and measured in a Gedanken experiment with a so-called virtual detector $[68,69]$. In both cases, time delay arises due to sub-barrier dynamics.

The asymptotic time delay is derived from the asymptotic photoelectron momentum distribution (PMD). It is defined by the classical backpropagation of the peak of the photoelectron asymptotic wave function up to the tunnel exit [43-46,70,71]. A theoretical description of the experimental asymptotic time delay is challenging because of the entanglement of Coulomb field effects with those of the tunneling delay. In a regime far from over-the-barrier ionization (OTBI), the asymptotic time delay is vanishing [36,37] However, in regimes approaching the OTBI the asymptotic delay is not negligible; it was in fact found to be negative [42-44] and shown to arise due to interference of direct and under-the-barrier recolliding trajectories [52].

In the tunneling region, the tunneling time delay is deduced by following the peak of the electron wave function, the so-called Wigner trajectory. A few de Broglie wavelengths away from the tunnel exit, the electron dynamics are quasiclassical and the classical trajectory is accurately 
described by the eikonal of the wave function in the WentzelKramers-Brillouin (WKB) approximation. However, near the tunnel exit, the WKB approximation fails and a full quantummechanical description is given by the Wigner trajectory. Near the tunnel exit this description diverges strongly from the classical one but nevertheless approaches it asymptotically, as one moves toward a detector. The Wigner time delay has been calculated numerically with the virtual detector method in Refs. [33-36], showing positive time delay and nonvanishing longitudinal velocity at the tunnel exit.

In this paper we investigate the Wigner time delay in tunneling ionization, which a virtual detector in a Gedanken experiment would observe. We concern ourselves chiefly with understanding the principles of the tunneling delay, and to this end we consider a simple, time-dependent model of a one-dimensional (1D) atom, with an electron bound by a short-range potential, which is ionized by a half-cycle laser pulse.

At first sight, such abstraction may seem overly simplistic; nonetheless, it takes into account all the necessary features of tunneling ionization. First, ionization occurs mainly in the direction of the electric field so a 1D treatment is appropriate. Likewise, no real laser pulse is a half-cycle sinusoid but by considering one such laser we ensure no continuum electron-to-atomic core recollisions take place, which convolute the physical picture. Such an interpretation is rendered clearer by having analytic expressions, unattainable with a pure Coulomb atomic potential but feasible with a short-range potential.

In this manner, the time-dependent wave function is described within the strong-field approximation (SFA). The components of the wave function which are reflected by the tunneling barrier are identified by analyzing the saddle points of the time integral of the wave function. We show that the interference of reflected and transmitted components of the wave function generates the Wigner time delay, while the same delay vanishes when the reflected components are neglected. Additionally, the scalings with respect to the laser field strength of the Wigner time delay and group velocity at the tunnel exit are derived and interpreted.

We work well below the OTBI regime, in what we term the deep tunneling regime. While in this regime the asymptotic tunneling time delay is vanishing we show that the Wigner time at the tunnel exit (calculated in the first-order SFA) is not only nonzero, but sizable, as already noted in Ref. [37]. This is in contrast to the asymptotic time delay, where the first-order SFA result is vanishing and the second-order SFA is required to obtain a nonvanishing asymptotic time delay (attributable to sub-barrier recollisions and interference of paths).

The structure of the paper is as follows: in Sec. II we introduce the basic ionization model, calculate the SFA timedependent wave function, and construct the Wigner trajectory from the latter. To elucidate our method of analyzing the contribution of reflections to the wave function, in Sec. III we consider a simpler, analytic, model of ionization: an electron in a constant electric field. The analyticity of this model allows us to relate the reflected components of the wave function to the contribution of the specific saddle points of the integral representation of the wave function. We apply this concept to the SFA wave function in Sec. IV, whereby we show that neglecting reflections results in a zero Wigner time delay. Lastly, in Sec. V, we summarize and interpret our results and discuss the implications of these on the interpretation of attosecond streaking experiments. For the reader unacquainted with strong-field physics, the Wigner time delay for scattering by a square potential barrier was previously analyzed in Refs. [7,72], where the significance of reflections to the time delay was alluded to. In Appendix A this problem is revisited and the role of reflections is explicitly presented. Atomic units (a.u.) are used exclusively throughout this paper.

\section{TIME DELAY IN STRONG-FIELD IONIZATION}

The Wigner time, developed for scattering problems $[10,11,13]$, considers the time taken for the peak of the wave packet to travel a given distance. For tunneling through a time-independent barrier, the Wigner time can be derived via the energy derivative of the phase of the tunneling wave function $\psi$ :

$$
\tau_{W}(x)=-i \frac{\partial}{\partial \mathcal{E}} \ln \frac{\psi(x)}{|\psi(x)|} .
$$

The peak of the ionized wave packet leads to the asymptotic formation of a well-defined peak in the attoclock PMD, which is subsequently measured and interpreted.

As the peak of the wave packet follows the trajectory $\tau_{W}(x)$, the Wigner group delay velocity of the wave packet can be defined:

$$
v_{W}(x)=\left(\frac{\partial \tau_{W}(x)}{\partial x}\right)^{-1} .
$$

When a monochromatic wave is incident on a finite potential barrier, the propagation inside the barrier is a superposition of an exponentially suppressed wave (transmission) with a growing exponential wave, namely, the reflection by the surface of the barrier (see, e.g., Appendix A). The goal of this paper is to establish the causal effect of wave-function refections inside the barrier on the time delay in strong-field ionization.

In order to do this, we calculate the time-dependent ionized electron wave function at the position where the electron appears in the continuum, thereby allowing us to deduce the peak of the wave packet with respect to time. The Wigner time delay can be calculated as the energy derivative of the phase of the wave function via Eq. (1) in a static field but in a time-dependent laser field this prescription fails as energy is not conserved. Correspondingly, we derive the Wigner time delay at the tunnel exit explicitly as the delay of the peak of the wave packet, rather than from the energy derivative of the phase of the wave function.

\section{A. The model}

Consider an electron initially bound in a 1D short-range potential:

$$
V(x)=-\kappa \delta(x)
$$

with a binding energy $I_{p}=\kappa^{2} / 2$, and with a corresponding ground-state wave function $\psi_{0}(x, t)=\sqrt{\kappa} \exp \left(-\kappa|x|+i I_{p} t\right)$.

We describe the generation of the peak of the ionized wave packet due to tunneling ionization near the tunnel exit. For this 
reason, we wish to avoid recollisions in the continuum and so a half-cycle laser field is employed in our model. The laser electric field,

$$
E(t)=-E_{0} \cos ^{2}(\omega t),
$$

is switched on at $\omega t_{0}=-\pi / 2$. In a half-cycle laser field the ionization happens mostly near the peak of the field. Here, nonadiabatic effects are governed by the second derivative of the field at the peak $E^{\prime \prime}(0) / E(0)=2 \omega^{2} \equiv \omega_{\text {eff }}^{2}$, where we define the effective frequency $\omega_{\text {eff }}=\sqrt{2} \omega$.

We consider the tunnel ionization regime and keep constant the Keldysh parameter [73]:

$$
\gamma=\sqrt{\frac{I_{p}}{2 U_{p}}} \ll 1
$$

where $U_{p}=E_{0}^{2} /\left(4 \omega_{\text {eff }}^{2}\right)$ is the ponderomotive potential. Thus, when varying the field strength $E_{0}$, we vary the frequency $\omega=$ $\gamma E_{0} /(\sqrt{2} \kappa)$ accordingly.

Moreover, we consider the so-called deep tunneling regime, wherein $E_{0} \ll E_{\mathrm{th}}$, and $E_{\mathrm{th}}$ is the threshold field of OTBI. This threshold can be estimated as the field strength where the coordinate-saddle point of the SFA-matrix element, i.e., the starting point $x_{s} \approx \sqrt{\kappa / E_{0}}$ of the quantum orbit, becomes comparable to the tunnel exit $x_{e}$, which for the shortrange potential corresponds to the condition $E_{\mathrm{th}} \approx \kappa^{3} / 4$ [74].

The superposition of the laser field with the atomic potential creates a potential barrier, through which an electron can tunnel, with a characteristic classical tunnel exit $x_{e}=I_{p} / E_{0}=$ 10 a.u. [75]. While this is a rudimentary estimate, it is in agreement with a tunnel exit derived quantum mechanically in our parameter regime as shown in Sec. IV B.

\section{B. Time evolution of the wave function}

Our investigation is based on the SFA wave function describing the electron dynamics in tunneling ionization. We define the Wigner trajectory and tunneling time delay employing the virtual detector approach $[34,35,68,69]$ based on the SFA wave function. The ionization dynamics are described by the Schrödinger equation

$$
i \frac{\partial}{\partial t} \Psi(x, t)=\left(H_{0}+H_{i}\right) \Psi(x, t),
$$

with the atomic Hamiltonian

$$
H_{0}=-\frac{1}{2} \frac{\partial^{2}}{\partial x^{2}}+V(x)
$$

and the interaction Hamiltonian with the laser field

$$
H_{i}=x E(t) .
$$

The standard SFA is employed for the solution of the Schrödinger equation. In the interaction picture with Hamiltonian $H=H_{0}+H_{i}$, the time-evolution operator $U\left(t, t_{0}\right)$ satisfies the Dyson integral equations [76]

$$
U\left(t, t_{0}\right)=U_{0}\left(t, t_{0}\right)-i \int_{t_{0}}^{t} d t^{\prime} U\left(t, t^{\prime}\right) H_{i}\left(t^{\prime}\right) U_{0}\left(t^{\prime}, t_{0}\right),
$$

where $U_{0}$ is the evolution operator corresponding to the Hamiltonian $H_{0}$. A perturbation series can be constructed by replacing the full evolution operator $U\left(t, t_{0}\right)$ in the Dyson integral with the evolution operator corresponding to the Hamiltonian $H_{i}$, namely,

$$
U_{f}\left(t, t^{\prime}\right)=\int d p\left|\Psi_{p}(t)\right\rangle\left\langle\Psi_{p}\left(t^{\prime}\right)\right|
$$

where $\left|\Psi_{p}\right\rangle$ are the Volkov states [77]. Each order in the perturbation theory corresponds to a sub-barrier recollision in our half-cycle laser field. As each sub-barrier recollision implies a longer tunneling path, each term in this series is suppressed by the Keldysh exponent $\exp \left(-2 \kappa^{3} / 3 E_{0}\right)$ and so, in contrast to the near OTBI regime, in the deep tunneling regime a first-order expansion in the SFA suffices (i.e., neglecting sub-barrier recollisions) [52].

Consequently, the electron state during the interaction takes the form $|\psi(t)\rangle=\left|\psi_{0}(t)\right\rangle+\left|\psi_{i}(t)\right\rangle$ where $\left|\psi_{0}(t)\right\rangle$ is the eigenstate of the atomic Hamiltonian $H_{0}$ and

$$
\left|\psi_{i}(t)\right\rangle=-i \int_{-\infty}^{\infty} d p \int_{t_{0}}^{t} d t^{\prime}\left|\Psi_{p}(t)\right\rangle\left\langle\Psi_{p}\left(t^{\prime}\right)\left|H_{i}\left(t^{\prime}\right)\right| \psi_{0}\left(t^{\prime}\right)\right\rangle
$$

describes the electron dynamics induced by the laser field, which generates the Wigner trajectory. It is the dominant part of the wave function at distances far from the core, and therefore, we concentrate on the analysis of its behavior. Denoting the electronic kinetic momentum as

$$
\mathcal{P}(t) \equiv p+A(t)
$$

with the laser vector potential $A(t)=-\int_{-t_{0}}^{t} E\left(t^{\prime}\right) d t^{\prime}$, the 1D Volkov state in the length gauge is

$$
\left|\Psi_{p}(t)\right\rangle=|\mathcal{P}(t)\rangle e^{-i S(t)}
$$

with a plane-wave component $\langle x \mid p\rangle=(2 \pi)^{-\frac{1}{2}} \exp (i p x)$ and the Volkov phase

$$
S(t)=\frac{1}{2} \int^{t} d \tau \mathcal{P}(\tau)^{2}
$$

We can expand the overlap in (11) using a resolution of identity in a dummy variable $x^{\prime}$ :

$$
\begin{aligned}
& \left\langle\Psi_{p}\left(t^{\prime}\right)\left|H_{i}\left(t^{\prime}\right)\right| \psi_{0}\left(t^{\prime}\right)\right\rangle \\
& =\frac{e^{i\left(I_{p} t^{\prime}-S\left(t^{\prime}\right)\right)}}{\sqrt{2 \pi}} E\left(t^{\prime}\right) \int_{-\infty}^{\infty} d x^{\prime} e^{i \mathcal{P}\left(t^{\prime}\right) x^{\prime}-\kappa\left|x^{\prime}\right|} x^{\prime} .
\end{aligned}
$$

While this integral is soluble, we do not evaluate it but rather first integrate (11) with respect to $p$ and then $x^{\prime}$. With the notation $\Delta f_{n}\left(t, t^{\prime}\right)=f_{n}(t)-f_{n}\left(t^{\prime}\right)$, and defining the integrals $f_{n}(t)=\int^{t} d t^{\prime} A\left(t^{\prime}\right)^{n}$, we may perform the initial integral over 


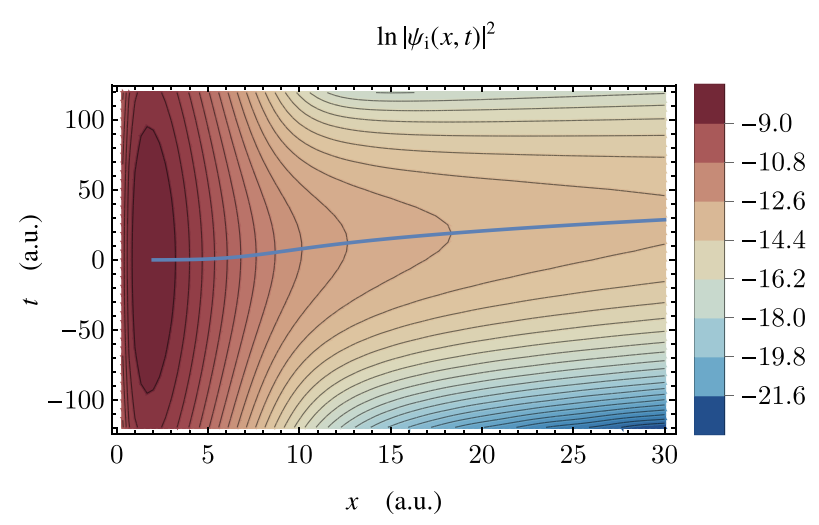

(a)

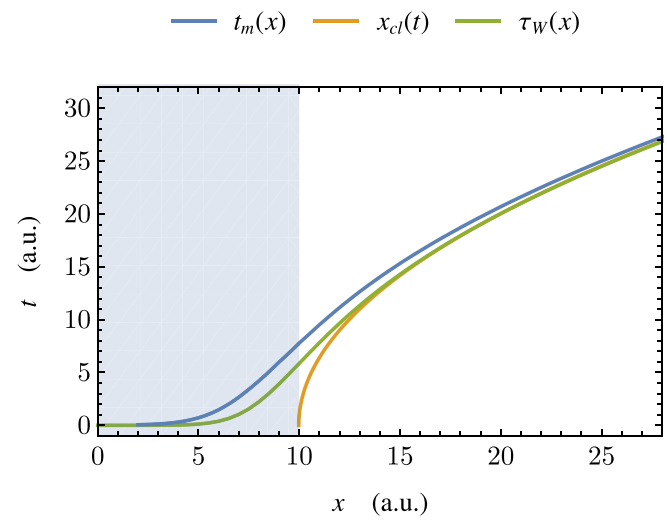

(b)

FIG. 1. (a) Ionization amplitude $\left|\psi_{i}(x, t)\right|^{2}$ as defined in Eq. (17). At every cross section in $x$, the temporal peak of the wave function was determined (plotted in blue) which can be interpreted as the trajectory of the peak. (b) Comparison of trajectories in the region near the tunnel exit, $x_{e}$. The displayed curves are the peak trajectory [blue, given by the maximum of $\left|\psi_{i}(x, t)\right|^{2}$ ], the classical trajectory (orange, given by the Newton equation starting at the tunnel exit), and the Wigner trajectory [green, given by Eq. (25)]. Under the barrier $(x<10$, shaded blue), there is an increasing delay of the peak with respect to the laser peak. Outside the barrier, the peak trajectory rapidly converges with the classical and Wigner electron trajectory. Near the atomic core $(x<2)$, the bound state, $\left|\psi_{0}(x)\right| \gg\left|\psi_{i}(x)\right|$, dominates the full wave function $\psi(x)=\psi_{0}(x)+\psi_{i}(x)$ so the meaning of a trajectory for ionization is lost.

$p$ which is a simple Gaussian integral. The resulting expression

$$
\psi_{i}(x, t)=\int_{t_{0}}^{t} d t^{\prime} \int_{-\infty}^{\infty} d x^{\prime} \frac{x^{\prime} \sqrt{\kappa} E\left(t^{\prime}\right)}{\sqrt{2 \pi i\left(t-t^{\prime}\right)}} \exp \left[i\left(I_{p} t^{\prime}+i \kappa\left|x^{\prime}\right|+x\left[A(t)-A\left(t^{\prime}\right)\right]+\frac{i\left[\left(x-x^{\prime}\right)-\Delta f_{1}\left(t, t^{\prime}\right)\right]^{2}}{2\left(t-t^{\prime}\right)}-\frac{\Delta f_{2}\left(t, t^{\prime}\right)}{2}\right)\right]
$$

may then be finally integrated in $x^{\prime}$ to yield

$$
\psi_{i}(x, t)=\int_{t_{0}}^{t} d t^{\prime} \sqrt{\kappa} E\left(t^{\prime}\right)\left(\frac{z_{-}^{2}\left[1+\operatorname{erf}\left(z_{-}\right)\right] e^{-z_{+}^{2}}}{z-\kappa}+\frac{z_{+}^{2}\left[1-\operatorname{erf}\left(z_{+}\right)\right] e^{-z_{-}^{2}}}{z+\kappa}\right) \exp (i \zeta) \equiv \int_{t_{0}}^{t} d t^{\prime} \exp \left[i \Phi\left(x, t, t^{\prime}\right)\right]
$$

where we have defined $z_{ \pm}=\sqrt{\frac{i}{2}\left(t-t^{\prime}\right)}(z \pm \kappa)$ for

$$
z=-i\left(A\left(t^{\prime}\right)+\frac{x-\Delta f_{1}\left(t, t^{\prime}\right)}{t-t^{\prime}}\right)
$$

and where

$$
\begin{gathered}
\zeta=I_{p} t^{\prime}+x A(t)+ \\
\left(t-t^{\prime}\right)\left(\frac{\left[x-\Delta f_{1}\left(t, t^{\prime}\right)\right]^{2}}{2\left(t-t^{\prime}\right)^{2}}+\kappa^{2}+z^{2}\right)-\frac{\Delta f_{2}\left(t, t^{\prime}\right)}{2} .
\end{gathered}
$$

Our aim is to calculate the wave function and Wigner time delay in the interaction region around the tunnel exit by studying the phase $\Phi$. Thus, in deviation to standard SFA studies, the time integral is calculated up to the finite observation time $t$, meaning the integration must be performed numerically. The usual saddle-point integration method is valid only for sufficiently large values of $t$, placing one well outside the tunneling region.

\section{Analysis}

For the numerical integration of the SFA wave function Eq. (17) an electric-field strength $E_{0}=0.05$ a.u. and Keldysh parameter $\gamma=\sqrt{2} / 10 \approx 0.14$ were chosen to ensure deep tunneling is considered. With these parameters, our half-cycle laser pulse spans $[-314,314]$ a.u. We present results for the case of hydrogen, for which $I_{p}=\kappa^{2} / 2=1 / 2$ a.u., implying an OTBI threshold field $E_{\text {th }} \approx 0.25$ a.u. The time integration was carried out with the standard numerical integration routine of MATHEMATICA 12 to a precision of 30 digits.

We build the Wigner trajectory from the partial wave function $\psi_{i}$, which in the SFA describes the electronic dynamics induced by the laser field during the tunneling ionization process. From this laser driven perturbation $\psi_{i}$, in regions further from the atomic core, the outgoing ionized wave emerges. Note, however, that in the interaction region close to the atomic core, the laser driven bound and free parts are inseparable. The space-time probability distribution, $\left|\psi_{i}(x, t)\right|^{2}$, is presented in Fig. 1. From this distribution the Wigner trajectory is derived as follows: the probability time distribution at each space point $x$ is invoked [see Fig. 2(a) for the case of the tunnel exit coordinate $x=x_{e}$ ] and the maximum $t_{m}(x)$ of this distribution is derived. The Wigner trajectory is represented by the function $t_{m}(x)$, which runs along all maximum points of the space-time distribution.

We underline that the wave function $\psi_{i}$ vanishes after the laser field is switched off. Thus, $\psi_{i}$ does not include the possible net excitations of the atomic states due to interaction with the laser field. This is a feature of the SFA, where the 


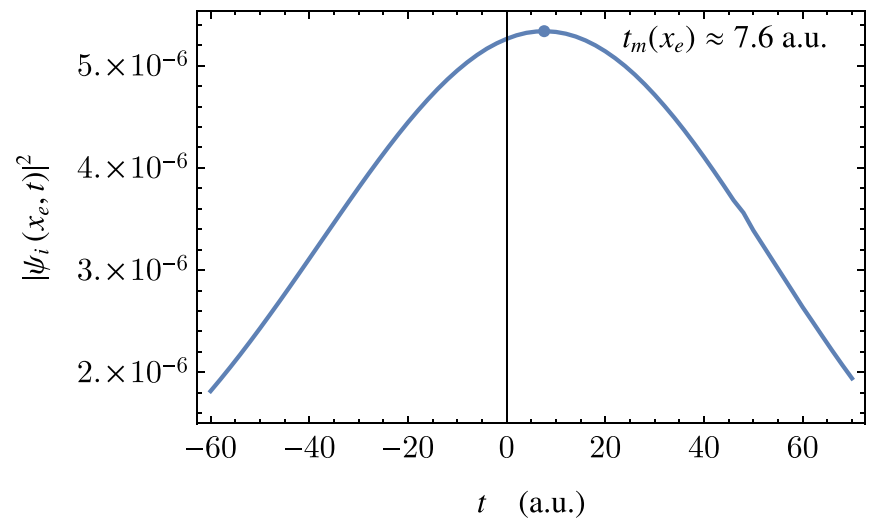

FIG. 2. Probability distribution $\left|\psi_{i}\left(x_{e}, t\right)\right|^{2}$ vs time at the classical tunnel exit $x_{e}=I_{p} / E_{0}$. This distribution is peaked at a greater time, $t_{m} \approx 7.6$ a.u. $\approx 183$ as, marked by the dot, than the peak of the laser pulse.

exact time-evolution operator in the final state is replaced by the Volkov propagator, representing the continuum electron in the laser field.

We note the maximum of the wave function displays a time delay with respect to the peak of the field. The tunneling time delay under the barrier $(x<10)$ increases when moving towards the tunnel exit. Outside the barrier, this trajectory rapidly approaches the classical electron trajectory (beginning at the classical tunnel exit with zero momentum). The slight deviation is due to quantum-mechanical corrections to the quasiclassical wave function not far from the tunnel exit.

The main idea advocated in this paper is that the Wigner time delay during tunneling is closely related to reflections arising during tunneling dynamics. It is straightforward in the simple case of tunneling through a box potential to show that reflections are responsible for the tunneling time delay, as is done in Appendix A. However, unlike in the separable box potential case, a given wave function is not easily deconstructible as a simple superposition of reflected and transmitted components corresponding to simple decaying or growing exponentials. Instead, the transmitted and reflected components are completely encapsulated in the wave function, making it rather more problematic to disentangle. This is ultimately achieved in Sec. V for the SFA wave function presented in this paper but in order to highlight the main aspects of the method we discuss beforehand a simpler example, that of tunneling in a constant electric field.

\section{TIME DELAY IN A CONSTANT FIELD}

The adiabatic model of ionization, namely, atomic ionization in a constant field, will be helpful for our purposes as it is analytically tractable. Moreover, it will provide us with a reference with which to compare our earlier time-dependent model.

\section{A. Ionization in an adiabatic field}

Consider a bound electron of energy $-I_{p}$ in $1 \mathrm{D} \delta$ potential ionized by a constant electric field $E_{0}$. The continuum eigenstate of the electron in this field is given by the solution to the time-independent Schrödinger equation:

$$
-\frac{1}{2} \frac{d^{2} \psi}{d x^{2}}+\left(I_{p}-E_{0} x\right) \psi(x)=0,
$$

which has as a general analytical solution a superposition of the Airy functions of the first and second kind:

$$
\psi(x)=c_{1} \operatorname{Ai}(\tilde{x})+c_{2} \operatorname{Bi}(\tilde{x})
$$

where

$$
\tilde{x}=\left(\frac{2}{E_{0}^{2}}\right)^{\frac{1}{3}}\left(I_{p}-E_{0} x\right) .
$$

The requirement that the wave function be a traveling wave as $x \rightarrow \infty$ imposes the condition $c_{1}=i c_{2}$ so that the wave function takes the form

$$
\psi(x)=T[\operatorname{Bi}(\tilde{x})+i \operatorname{Ai}(\tilde{x})],
$$

where $T$ can be determined by matching this wave function to the bound-state solution of the atom; this prefactor plays a role in the ionization amplitudes but is irrelevant to the phase of the wave function. We calculate the Wigner time delay for this wave function via the energy derivative of the phase:

$$
\tau_{W}(x)=i \frac{\partial}{\partial I_{p}} \ln \frac{\psi}{|\psi|}=\frac{2^{\frac{1}{3}}}{\pi E_{0}^{\frac{2}{3}}} \frac{1}{\operatorname{Ai}(\tilde{x})^{2}+\operatorname{Bi}(\tilde{x})^{2}} .
$$

The constant field model allows one to estimate the scaling of the Wigner time delay, given by Eq. (1), at the tunnel exit $x_{e}=I_{p} / E_{0}$ :

$$
\tau_{W}\left(x_{e}\right)=\frac{3^{4 / 3} \Gamma\left(\frac{2}{3}\right)^{2}}{2^{5 / 3} \pi} \frac{1}{E_{0}^{2 / 3}} .
$$

Using Eq. (2), we derive the scaling of the Wigner group velocity of the electron at the tunnel exit,

$$
v_{W}\left(x_{e}\right)=\frac{2\left(\frac{2}{3}\right)^{2 / 3} \sqrt{\pi} \Gamma\left(\frac{7}{6}\right)}{\Gamma\left(\frac{2}{3}\right)^{2}} E_{0}^{1 / 3},
$$

and find it consistent with the estimate of the scaling of the electron momentum $p_{e} \sim E_{0} \tau_{W}$ in Ref. [52].

We compare the Wigner time delay and the group delay velocity in the constant field case with the exact time-dependent SFA calculations in Fig. 3. The time delay in the SFA scales with the electric field in a similar manner to the adiabatic case, $\tau_{W}\left(x_{e}\right) \propto 1 / E_{0}^{2 / 3}$, but shifted in time by a constant. This shift stems from the fact that the Wigner trajectory is derived via the energy derivative of the phase of the wave function (which corresponds to following the peak of the wave packet, neglecting its spreading), while the time integration responsible for the formation of the ionization wave packet in SFA is calculated exactly numerically.

The scaling of the Wigner group velocity using the SFA is also in agreement with the adiabatic estimate, $v_{W}\left(x_{e}\right) \propto E_{0}^{1 / 3}$. However, the relative error between these two grows with the field strength, becoming appreciable at higher field strengths when approaching the OTBI threshold. This is consistent with the simple estimate for the threshold for OTBI, $E_{\mathrm{th}} \approx$ 0.25 a.u., where a tunneling description no longer applies. 


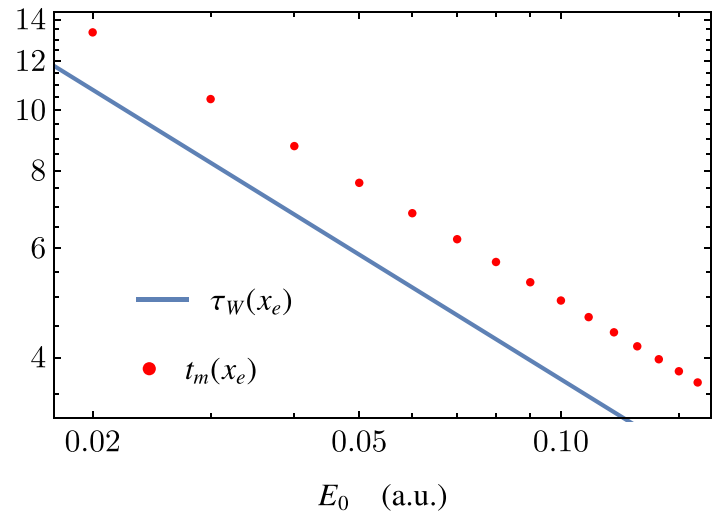

(a)

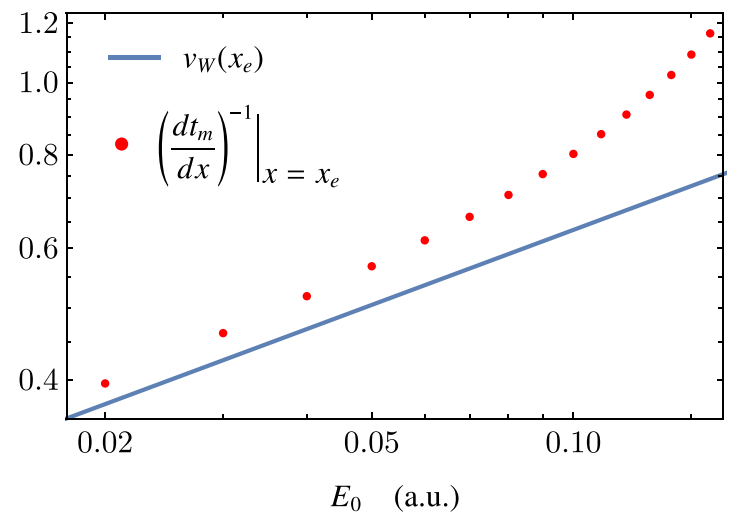

(b)

FIG. 3. Log-log plots of the scaling with respect to field strength, $E_{0}$, of the (a) Wigner time delay and (b) group velocity at the classical tunnel exit, $x_{e}=I_{p} / E_{0}$, for the time evolved SFA wave function (red dots) and the adiabatic constant field wave function (blue lines). For the time evolved wave function, the maxima in time, $t_{m}$, of the probability distribution $\left|\psi\left(x_{e}, t\right)\right|^{2}$ and its derivative were calculated; for the adiabatic case, results follow from Eqs. (26) and (27). The agreement in the trends is good, deteriorating as one approaches the OTBI threshold $E_{\text {th }} \approx 0.25$ a.u.

Note that the Wigner trajectory in the constant field case is determined from the solution of the time-independent Schrödinger equation (energy eigenstate), while in the SFA case it is determined from the time-dependent wave function. In the first case, the Wigner trajectory is defined as the derivative of the wave-function phase with respect to the energy, which corresponds to determining the coordinate of the peak of the electron wave packet at a fixed time moment, i.e., determining the motion of the wave-packet peak. In contrast, in the SFA case we explicitly determine the maximum of the wave function in time for a fixed coordinate.

\section{B. Reflections in a constant field}

To investigate the effect of under-the-barrier reflected components, we need to isolate their contribution to the full wave function. The analytical wave function of Eq. (24) consists of a superposition of Airy functions $\operatorname{Ai}(\tilde{x})$ and $\operatorname{Bi}(\tilde{x})$ which can be given interpretation as the under-the-barrier reflected and transmitted components of the tunneling wave function, respectively.

This interpretation can be generally understood by considering the Airy functions, as shown in Fig. 4. The Bi component of the wave function decays exponentially as it approaches the tunnel exit, $x=x_{e}$, from the atomic core at $x=0$, and hence can be seen to correspond to the transmission component; likewise, the exponentially growing $\mathrm{Ai}$ component corresponds to wave-function reflection.

This interpretation can be formally established by considering the integral representation of the Airy functions:

$$
\begin{aligned}
\operatorname{Ai}(\tilde{x}) & =\frac{1}{2 \pi i} \int_{\gamma_{1}} d s \exp \left(\tilde{x} s-\frac{s^{3}}{3}\right), \\
\operatorname{Bi}(\tilde{x}) & =\frac{1}{2 \pi} \int_{\gamma_{2}-\gamma_{3}} d s \exp \left(\tilde{x} s-\frac{s^{3}}{3}\right),
\end{aligned}
$$

where the complex plane integration paths $\gamma_{i}$ are indicated in Fig. 5(a). These integrals converge when their end points lie in the slices of the complex plane defined by $-\frac{\pi}{6}<\theta<\frac{\pi}{6}$, $\frac{\pi}{2}<\theta<\frac{5 \pi}{6}$, and $\frac{7 \pi}{6}<\theta<\frac{3 \pi}{2}$, where in polar $\{r, \theta\}$ coordinates $s=r \exp (i \theta)$. In these regions, shaded blue in Fig. 5, the integrand vanishes rapidly as $r \rightarrow \infty$.

The majority of the contributions to the Airy integrals thus come from around the saddle points,

$$
s_{ \pm}= \pm \sqrt{\tilde{x}},
$$

of the argument of the integrand. The Airy contours, $\gamma_{i}$, can be deformed into paths that to go through these saddle points and, using the standard technique of saddle-point integration method [78], asymptotic expressions for the Airy integrals can be determined.

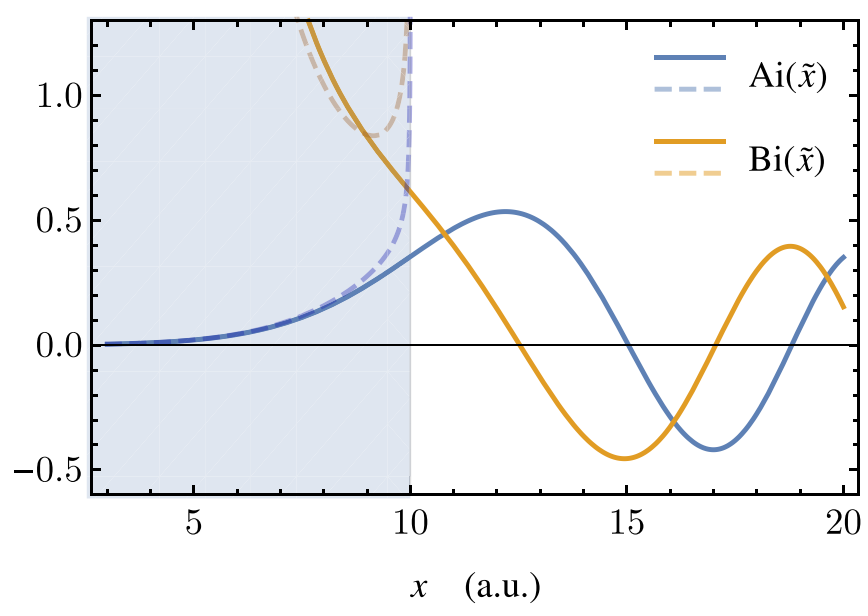

FIG. 4. The solution to the electron in a constant field problem is given by a superposition of Airy functions of the first and second kind, $\operatorname{Ai}(\tilde{x})$ and $\operatorname{Bi}(\tilde{x})$, plotted in blue and orange, respectively. These functions have very accurate asymptotic expansions, shown dashed, which can be derived by considering the saddle points of the Airy integrals (28) and (29). Under the barrier, $x<10$ (shaded blue), these expansions show that the wave-function components $\operatorname{Ai}(\tilde{x})$ and $\operatorname{Bi}(\tilde{x})$, respectively, correspond to the reflected and transmitted components of the wave function. 


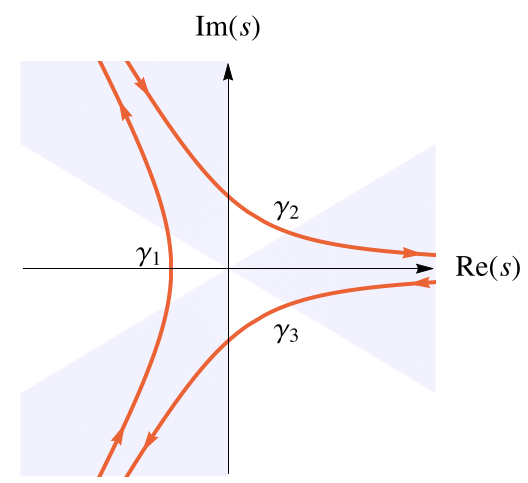

(a)

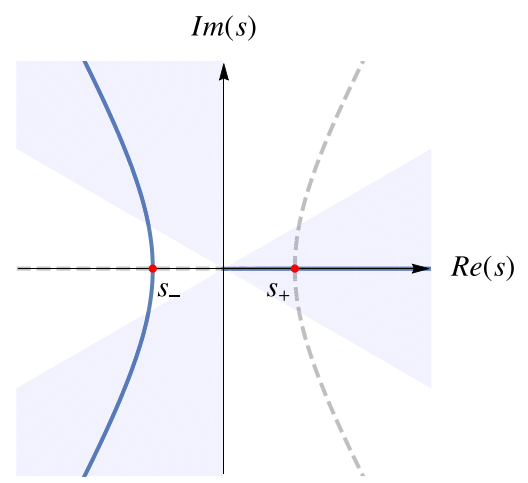

(b)

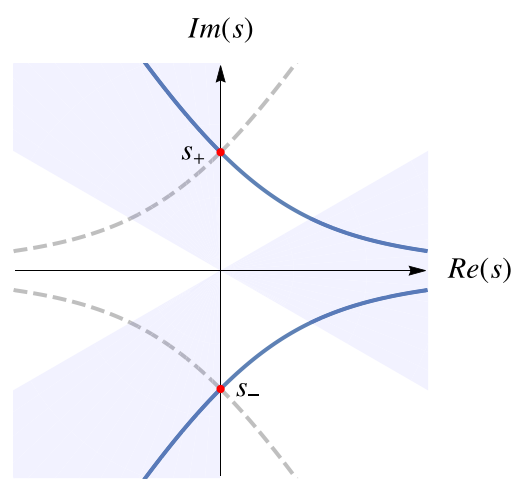

(c)

FIG. 5. The Airy integral $\int_{\gamma} d s \exp \left(\tilde{x} s-s^{3} / 3\right)$ is defined on the complex $s$ plane; it converges when the end points of its infinite contour, $\gamma$, lie in the shaded areas. The canonical contours defining the Airy function of the first $\left(\gamma_{1}\right)$ and second $\left(\gamma_{2}-\gamma_{3}\right)$ kind are shown in (a). Contributions to the Airy integral arise principally from portions of the contour near the saddle points of the integrand function $\exp \left(\tilde{x} s-s^{3} / 3\right)$. The configuration of the saddles $s_{ \pm}(x)= \pm \sqrt{\tilde{x}}$ (red dots) in the complex plane is shown for positions (b) inside the barrier $(\tilde{x}>0)$ and (c) outside the barrier $(\tilde{x}<0)$. Through each saddle point pass two perpendicular level curves, the path of steepest descents (solid blue) and the path of steepest ascents (dashed gray). Deforming the defining contours in (a) through the steepest descents contours in (b) and (c) provides asymptotic expansions for the Airy function, as in Eqs. (31) and (32).

The saddle points, and the respective paths of steepest descents and ascents, are illustrated in Figs. 5(b) and 5(c); since these are dependent on $\tilde{x}$ the application of the saddle-point method is different for the two cases of inside $(\tilde{x}>0)$ and outside $(\tilde{x}<0)$ the potential barrier. As shown in Fig. 5(b), for $\tilde{x}>0$ we may deform the contour $\gamma_{1}$ smoothly into the path of steepest descents for the saddle point $s_{-}$and in doing so obtain the asymptotic approximation

$$
\operatorname{Ai}(\tilde{x})=\frac{\exp \left(-\frac{2}{3} \tilde{x}^{\frac{3}{2}}\right)}{2 \sqrt{\pi} \tilde{x}^{\frac{1}{4}}}+O\left(\tilde{x}^{-\frac{3}{2}}\right)
$$

Likewise, we may deform the contours $\gamma_{2}$ and $-\gamma_{3}$ to both go through the steepest descent path of the saddle point $s_{+}$and hence deduce the asymptotic form of the $\operatorname{Bi}(x)$ function under the potential barrier:

$$
\operatorname{Bi}(\tilde{x})=\frac{\exp \left(+\frac{2}{3} \tilde{x}^{\frac{3}{2}}\right)}{\sqrt{\pi} \tilde{x}^{\frac{1}{4}}}+O\left(\tilde{x}^{-\frac{3}{2}}\right) .
$$

From a tunneling particle's perspective, one may identify the $\operatorname{Ai}(x)$ function and its saddle point $s_{-}$with the reflected part of the wave function, and the saddle point $s_{+}$, or the $\operatorname{Bi}(x)$ function with the transmitted part of the wave function: the transmitted wave decays as it moves to the tunnel exit, while the reflected wave propagates from the exit decaying exponentially toward the atomic core, as shown in Fig. 4.

In the following section, this relation between sub-barrier reflections and the contribution of one saddle point to the time integrand of the wave-function amplitude is applied to the problem of ionization in a time-dependent field. This then allows us to investigate the role of sub-barrier reflection in the formation of a tunneling time delay.

\section{WIGNER TIME AND REFLECTIONS IN THE SFA}

As shown in Sec. III, we may reasonably ascribe the saddle point of the integrand of the wave function to under-the- barrier reflection or transmission. To reveal the contribution of the reflection to the tunneling time delay, we investigate the complex continuation of the integrand function of the SFA wave function, Eq. (17). The insight developed previously, namely, the relation of a specific saddle point of the integrand function to reflectionlike behavior in the wave function, is used to extract the contribution of reflections to the wave function by modifying the path of integration of the complex plane.

In general, the complex $t^{\prime}$-plane picture of the phase $\Phi$ in Eq. (17) has many similarities to that of adiabatic ionization. However, it depends continuously not only on the coordinate $x$ but now also on observation time $t$, making its analysis somewhat more involved. In adiabatic ionization, there was only one degree of freedom, $\tilde{x}$, and the saddle points were either purely real or imaginary.

As shown in Fig. 6, in the time-dependent case there are also two saddle points of relevance in the SFA integral denoted $t_{ \pm}$. Their arrangement in the complex $t^{\prime}$ plane is dependent on $x$ and $t$ but, as in the adiabatic case, the real coordinate $x$ determines whether they are vertically or horizontally aligned (in or outside the barrier); the observation time $t$ merely shifts the axis of symmetry which is always observed around the line $t^{\prime}=t$. A more detailed discussion of the configuration of saddle points in $(x, t)$ parameter space may be found in Appendix B.

\section{A. Extracting reflections}

In the adiabatic case, a partial integration over only the contour containing the saddle point $s_{-}$would exclude the exponentially growing contributions to the wave function, i.e., the reflections, associated with the saddle point $s_{+}$.

We use the same principle in the time-dependent case; in Fig. 6 we identify the saddle point corresponding to reflections, denoted $t_{+}$(represented by a circle), and the secondary saddle point $t_{-}$(represented by a triangle). When we consider 


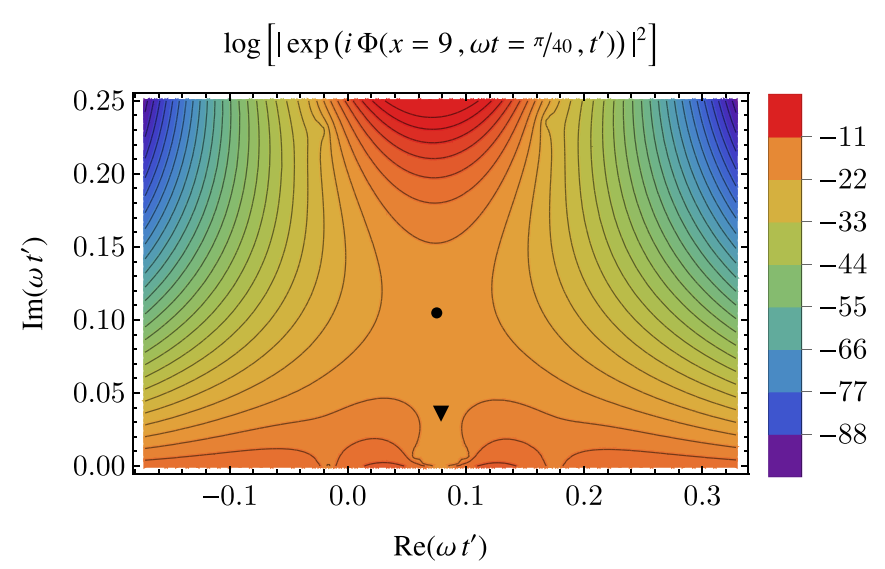

FIG. 6. Complex $t^{\prime}$ plane of the argument $\Phi\left(x, t, t^{\prime}\right)$ of the wavefunction integral $\psi_{i}=\int_{t_{0}}^{t} d t^{\prime} \exp (i \Phi)$, for parameters $x=9$ and $\omega t=\pi / 40$. For these configurations there are two saddle points, denoted by a circle and triangle, the former of which, $t_{+}$, corresponds to reflections. The saddle-point method can be used to solve the wave-function integral, when the path is taken over both saddle points. We use a partial path given by (33) to remove the contribution of reflections by integrating over only one of the saddle points. Other possible configurations of the saddle points are shown in Fig. 11.

the wave function under the potential barrier, we can split the integration contour in Eq. (17) into two parts: from the start of the pulse, $t_{0}=-\pi /(2 \omega)$, to the upper saddle point, $t_{+}$, and from $t_{+}$to the real time $t$.

The contributions of these two contours to the wave function are shown in Fig. 7. The former integral has the form of a growing exponential and so can ostensibly be identified as the reflected part of the wave function while the latter is a decaying exponential identifiable as the transmitted part. To neglect contributions from the reflections to the full wave

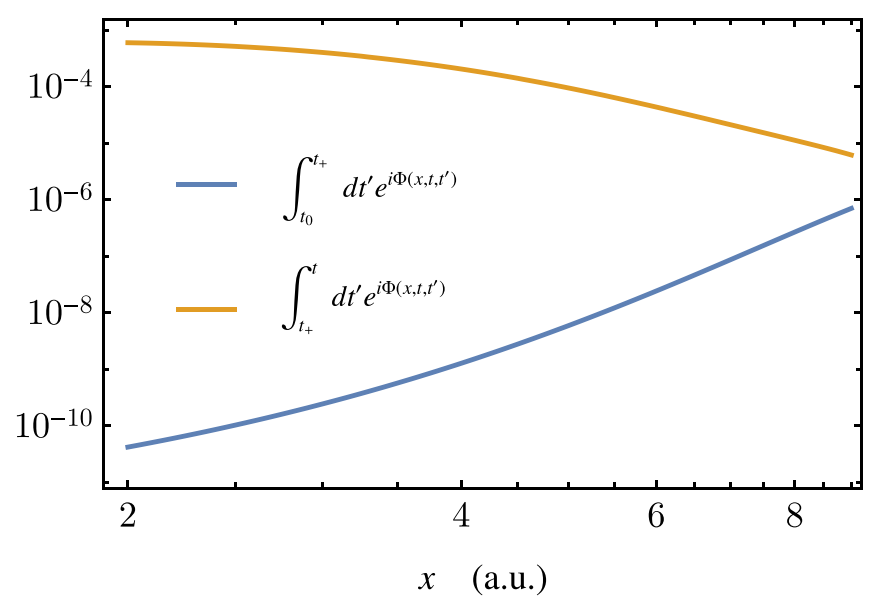

FIG. 7. Log-log plot of the relative contributions to the wavefunction amplitude from the portion of the integration path up to the saddle point (blue) and from the saddle point to the given real time $t$ (orange) for various positions $x$ under the classical barrier (i.e., far from the atomic core, but smaller than the tunnel exit). The behaviors are approximately decaying and growing exponentials, respectively, analogous to transmitted and reflected parts of the wave function. function we identify the following wave function:

$$
\psi_{\mathrm{nr}}(x, t)=\left\{\begin{array}{ll}
\int_{t_{0}}^{t_{+}} d t^{\prime} \exp \left[i \Phi\left(x, t, t^{\prime}\right)\right] & x>x_{e} \\
\int_{t_{+}}^{t} d t^{\prime} \exp \left[i \Phi\left(x, t, t^{\prime}\right)\right] & x<x_{e}
\end{array} .\right.
$$

Our method of distinguishing the reflection contribution is based on the distinguishability of the saddle-point contributions to the wave-function integral. This is not possible when the two saddle points are so close that the cubic term in the expansion of the phase $\Phi\left(x, t, t^{\prime}\right)$ becomes non-negligible [79], i.e., when

$$
\left|\frac{\frac{\partial^{3}}{\partial t^{\prime 3}} \Phi\left(x, t, t^{\prime}\right)}{\left(\frac{\partial^{2}}{\partial t^{\prime 2}} \Phi\left(x, t, t^{\prime}\right)\right)^{\frac{3}{2}}}\right|_{t^{\prime}=t_{ \pm}} \gtrsim 1
$$

for either saddle point $t_{ \pm}$. This occurs in the region very near the barrier boundary and at the peak of the laser pulse, as discussed further in Sec. IV B. In this case, the reflection cannot be separated in the wave function in a meaningful manner.

We find that when reflections are extracted from the wave function using Eq. (33) the Wigner time delay under the barrier vanishes, as evident in both panels of Fig. 8. That is, when reflections are neglected, there is no time delay between the peak of the electron probability distribution and the peak of the laser field. Moreover, outside the barrier, the trajectory of the peak rapidly becomes classical.

Plotted also in Fig. 8(b) is the trajectory for an electron in a constant field, given by Eq. (25). For parameters in the deep tunneling regime, the SFA trajectory (given by the peak of the wave function), and the classical trajectory, the constant field trajectory displays very similar behavior as the pulse elapses (the trajectories do not completely coincide far away due to the time dependence of the field and the need to match a tunnel exit, respectively).

At a detector infinitely far away such as one in an attoclock experiment, a signature of time delay would be absent for the deep tunneling conditions detailed in this paper. Thus, it is of importance to distinguish between a time delay of the peak of the wave function near the tunnel exit and an asymptotic time delay at a detector. Since in attoclock experiments the asymptotic momentum distribution is measured, i.e., the asymptotic time delay, one expects to find a zero time delay in the deep tunneling regime. However, near the tunnel exit, quantum-mechanical considerations must be taken into account and a quantum-mechanical treatment such as the one exposed here exhibits an explicit nonzero Wigner delay.

\section{B. Tunnel exit from the SFA}

We may also use the saddle-point analysis of Eq. (17) to extract relevant physical information about the electron dynamics. In particular, and in analogy to the constant field scenario, the topology of the saddle points through the complex $t^{\prime}$ plane reveals the functional behavior of the electronic wave packet.

The paths of these two saddle points, for two representative choices of time $t$ while varying $x$, are shown in Fig. 9, closely resembling those corresponding to Figs. 5(b) and 5(c). Much as in the constant field case, where the saddles are purely 


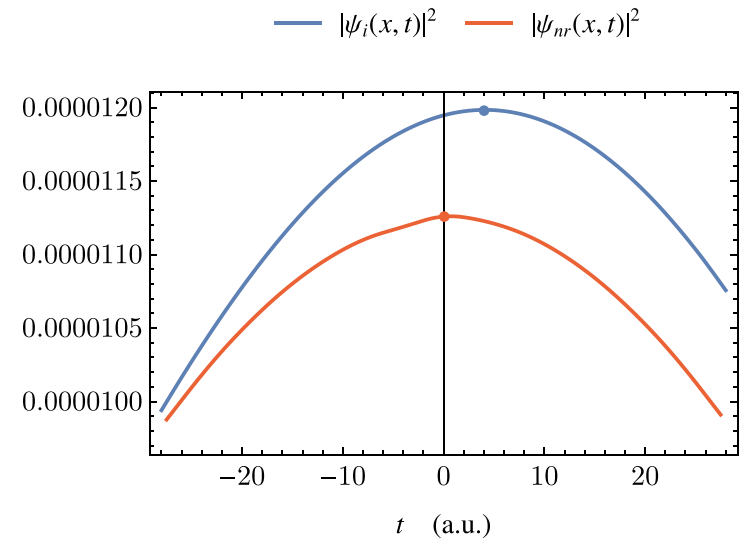

(a)

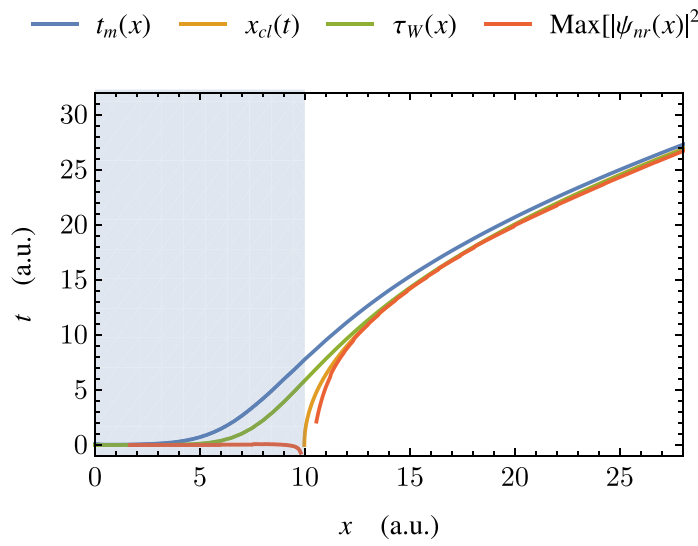

(b)

FIG. 8. (a) Temporal probability distributions at the position $x=8$ a.u. (under the barrier, near the tunnel exit), for the ionized wave function, $\psi_{i}$, and the pseudo-wave-function neglecting reflections, $\psi_{\mathrm{nr}}$. The physical wave function has accrued a Wigner time delay with respect to the laser field, whereas the maximum of the pseudo-wave-function is synchronous with the laser field peak. (b) Trajectories in the $(x, t)$ plane via the maximum of the wave function $\psi_{i}$ [given by $t_{m}(x)$ ], the maximum of the wave function neglecting reflections $\psi_{\mathrm{nr}}$, and the energy derivative of the constant field wave function $\tau_{W}(x)$. The classical electron trajectory, $x_{\mathrm{cl}}(t)$, starting at the tunnel exit at the peak of the laser field is also shown. Under the barrier, the probability distribution neglecting reflections $\left|\psi_{\mathrm{nr}}\right|^{2}$ shows zero time delay. In regions where Eq. (34) does not apply, mostly near the classical tunnel exit, we may not identify reflections or plot a subsequent trajectory.

real or imaginary, as given by Eq. (30) and Fig. 5, the pair of saddle points in the SFA is aligned around the line given by $\operatorname{Re}\left[t^{\prime}\right]=t$; this alignment is either vertical or horizontal depending on the coordinate $x$. For a fixed observation time $t$, there corresponds a coordinate $x$ of closest approach between the saddle points. For the peak of the pulse, $t=0$, there exists a coordinate $x_{t}$ where the saddle points merge completely, as they do in the case $\tilde{x}=0$ for the constant field electron.

Thus, two lines, $t=0$ and $x=x_{t}$, define separatrices for the behavior of the saddle points and their contributions to the integral: the behavior of the wave function can be seen to

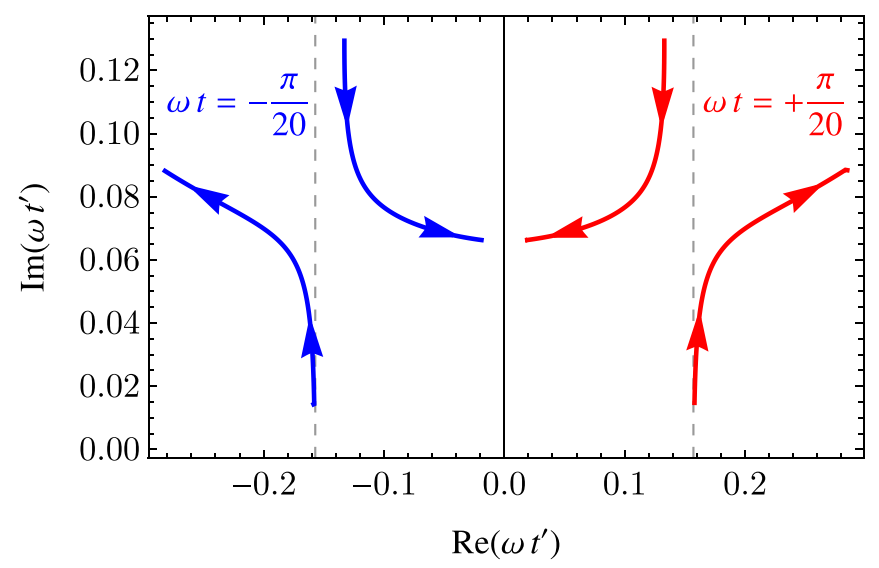

FIG. 9. Positions of the upper and lower saddle points of the integral (17) in the complex $t^{\prime}$ plane with varying $x$ and for fixed $\omega t=$ $\pm 0.1 \pi / 2$ (blue and red, respectively). The saddles draw smooth curves with varying $x$, where the arrows indicate growing values of $x$. For values of $x \lesssim 7$, the lower saddle point disappears below the imaginary axis. Each pair of curves is centered around the line $\operatorname{Re}\left(\omega t^{\prime}\right)=\omega t$, shown in dashed line. For the case $\omega t=0$, not shown, the two lines meet one point corresponding to $x=x_{t}$. change depending on whether $x>x_{t}$. This is a phenomenon exactly analogous to the merging of the saddle points for the constant field case of Sec. III: there, the merging of the saddle points occurs at the classical exit, $\tilde{x}=0$, and separates the regions of evanescence and oscillation in the wave function.

We may use this parallel to identify the merging point as a measure of the tunnel exit. In our paper, for a field strength of $E_{0}=0.05$ and $\gamma=0.14$, we find that this exit takes the value $x_{t} \approx 10.2$ a.u.; this is in good agreement with the classically expected tunnel exit $x_{e}=I_{p} / E_{0}=10$ a.u. More precise agreement is achieved with the probability averaged tunnel exit:

$$
x_{K}=\frac{\int d t^{\prime} \frac{I_{p}}{\left|E\left(t^{\prime}\right)\right|} \exp \left(-\frac{2 \kappa^{3}}{3\left|E\left(t^{\prime}\right)\right|}\right)}{\int d t^{\prime} \exp \left(-\frac{2 \kappa^{3}}{3\left|E\left(t^{\prime}\right)\right|}\right)} \approx 10.35 \text { a.u. }
$$

where the integration runs over the whole laser pulse.

\section{CONCLUSIONS}

In this paper, we have analyzed the tunneling time delay in strong-field ionization for a simple model of an atom with a short-range potential. The wave function was calculated to first order in the SFA for any intermediate time, enabling a quantum treatment of dynamics in the region where quasiclassical descriptions break down, viz., around the classical tunnel exit. For a given coordinate, the peak of the wave packet shows a time delay with respect to the peak of the laser field and this time delay is positive at the classical tunnel exit.

We argue that reflections of the electron wave packet under the tunneling barrier are fundamentally responsible for this nonzero time delay around the tunnel exit. This is a general phenomenon, present in any regime of strong-field ionization, as well as in any tunneling process. In particular, a simple showcase is presented in Appendix A for the case of tunneling through a box potential. 
To identify and separate contributions of reflected components to the wave function, we first considered the analytically soluble case of an electron in a constant electric field. In addition to providing a benchmark to our SFA wave function, the constant field wave function can be deconstructed by analyzing its definition as an integral in the complex plane; this integral is dominated by two saddle points, allowing us to unambiguously identify the transmitted and reflected wavefunction components as contributions to the integral from the regions around each saddle point.

We apply this observation to the SFA wave function by defining an integration contour that purposefully neglects the contribution of the reflection saddle point, and obtain a pseudo-wave-function distribution that has zero time delay with respect to the peak of the laser field everywhere under the barrier. Moreover, the tunnel exit is unequivocally determined from the SFA theory as the spatial co-ordinate at which the saddle points merge at the peak of the laser field.

Finally, we emphasize the distinction between the time delay present at the tunnel exit (theoretically measurable using a virtual detector) and an (experimentally observed) asymptotic delay. We have shown within the first-order SFA that the signature of the time delay in the electron wave function vanishes since the ionized electron propagates to the detector as the classical (i.e., with zero time delay) and the quantum-mechanical electron share equivalent asymptotic trajectories. Thus, the asymptotic time delay associated with the direct ionization path is always vanishing. Nevertheless, a more accurate estimate of the asymptotic time delay via the second-order SFA in Ref. [52] has shown that interference of the direct and the sub-barrier ionization paths generates nonvanishing negative asymptotic time delay in the near threshold of the OTBI regime, which fades out in the deep tunneling regime.

\section{ACKNOWLEDGMENTS}

The authors are grateful to the referees for their useful remarks, which generated fruitful discussions. This paper includes parts of the Ph.D. thesis work of Daniel Bakucz Canário, submitted to Heidelberg University, Germany.

\section{APPENDIX A: TUNNELING THROUGH A BOX POTENTIAL BARRIER}

In this Appendix we consider the role of quantum reflections in the tunneling time delay for an electron wave packet tunneling through a one-dimensional box potential. This is perhaps the simplest example of tunneling time delay since the wave function is readily separable (and of analytic solution) in the regions inside and outside the barrier, allowing the contributions of reflections to the time delay to be clearly identified.

It should be mentioned that this model differs from true strong-field ionization in that it considers the scattering of a wave packet incident on a potential barrier; in actual ionization the electron originates from within a potential barrier. Be that as it may, this paper provides a simple, intuitive picture of tunneling time delay, suitable even for the uninitiated.

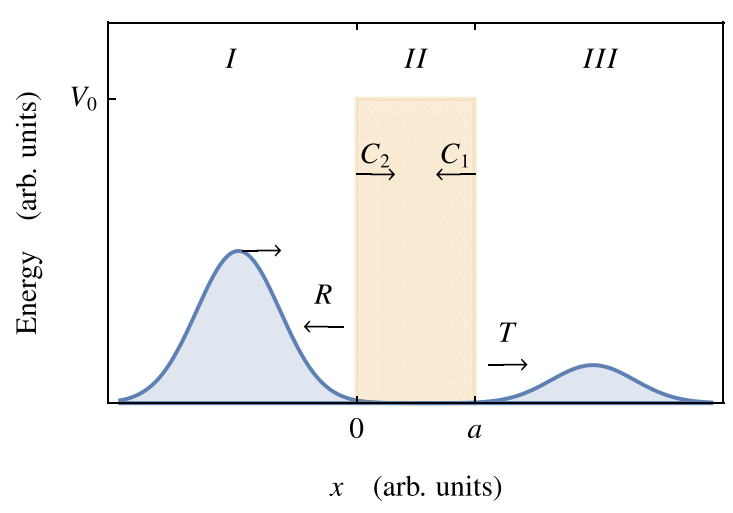

FIG. 10. Pictorial representation of the square barrier potential for an incident monochromatic wave. The wave function is a piecewise solution of the Schrödinger equation for the three regions shown, given by Eqs. (A2) and (A3).

\section{The box potential}

Consider a wave packet

$$
\Psi(x, t)=\int d p f\left(p-p_{0}\right) \psi(p) e^{-i \mathcal{E}(p) t}
$$

with energy $\mathcal{E}(p)=p^{2} / 2$, incident on a potential barrier $V(x)=V_{0}$ for $0 \leqslant x \leqslant a$ and zero elsewhere, where $f\left(p-p_{0}\right)$ is some distribution peaked at $p_{0}$ (e.g., a Gaussian), as shown Fig. 10. Each $p$-component wave function obeys the time-independent Schrödinger equation with the piecewise solution

$$
\begin{gathered}
\psi_{\mathrm{I}}(x)=e^{i p x}+R e^{-i p x}, \\
\psi_{\mathrm{II}}(x)=C_{1} e^{q x}+C_{2} e^{-q x}, \\
\psi_{\mathrm{III}}(x)=T e^{i p x},
\end{gathered}
$$

with momenta $p=\sqrt{2 \mathcal{E}}, q=\sqrt{2\left(V_{0}-\mathcal{E}\right)}$. The amplitude of the incoming wave has been set to unity, without loss of generality, and the coefficients $C_{1}$ and $C_{2}$ are the typical reflection and transmission coefficients under the barrier, respectively.

Matching the above solutions and their derivatives at the boundaries yields the coefficients

$$
\begin{aligned}
C_{1} & =\frac{(-2 i \chi)(1+i \chi) e^{-\xi}}{(1-i \chi)^{2} e^{\xi}-(1+i \chi)^{2} e^{-\xi}}, \\
C_{2} & =\frac{(-2 i \chi)(1-i \chi) e^{\xi}}{(1-i \chi)^{2} e^{\xi}-(1+i \chi)^{2} e^{-\xi}}, \\
R & =\frac{\left(1+\chi^{2}\right)\left(e^{-\xi}-e^{\xi}\right)}{(1-i \chi)^{2} e^{\xi}-(1+i \chi)^{2} e^{-\xi}},
\end{aligned}
$$

and

$$
T=\frac{(-4 i \chi) e^{-i p a}}{(1-i \chi)^{2} e^{\xi}-(1+i \chi)^{2} e^{-\xi}} .
$$

We have introduced the dimensionless parameters $\chi=p / q$ and $\xi=q a$ which, loosely speaking, determine the relative length and height of the barrier, respectively. 


\section{Time delay}

The wave packet after transmission is of the form

$$
\Psi_{\mathrm{III}}=\int d p|T(p)| \exp \{i[\varphi(p)+p x-\mathcal{E}(p) t]\},
$$

where $T=|T| e^{i \varphi}$. The maximum of this amplitude occurs when the phase in Eq. (A9) vanishes, that is, when (after some rearrangement)

$$
x=p_{0} t-\left[\frac{\partial \varphi}{\partial p}\right]_{p=p_{0}} .
$$

In the absence of a potential barrier, the peak travels at the classical velocity (in atomic units) $p_{0}$. Equation (A10) shows that the barrier causes a delay of the peak in reaching a given position $x$, a delay which is given by the energy derivative of the transmission phase $\varphi$. Thus,

$$
\tau=\frac{1}{p_{0}}\left[\frac{\partial \varphi}{\partial p}\right]_{p=p_{0}}+\frac{a}{p_{0}}
$$

gives the time delay of the peak after crossing the barrier, i.e., the tunneling time. For the box potential, one finds

$$
\tau=\frac{a}{p_{0}} \frac{\frac{1}{2 \xi}\left(\chi+\frac{1}{\chi}\right)^{2} \tanh (\xi)+\frac{\left(1-\chi^{2}\right)}{2} \operatorname{sech}^{2}(\xi)}{1+\frac{1}{4}\left(\chi-\frac{1}{\chi}\right)^{2} \tanh ^{2}(\xi)} .
$$

This can be expanded for large $\xi$ :

$$
\tau \approx \frac{a}{p_{0}}\left(\frac{2}{\xi}-\frac{8 e^{-2 \xi} \chi^{2}\left(\chi^{2}-1\right)}{\left(\chi^{2}+1\right)^{2}}\right),
$$

where the leading-order term can be identified as stemming from single reflections from the edges of the barrier, and the next-order term can be identified as the delay due to interference of single and double reflections from the barrier edges (i.e., waves that recoil twice before exiting). Higherorder terms correspond to interference of reflections at higher orders.

This is identical to the interference of direct and recolliding trajectories as described in Ref. [52]. Note that the latter is negative in the near-threshold tunneling regime of large $\chi$ and scales as a tunneling exponent $\propto e^{-2 \xi}$. The main body of the present paper is concerned with a single-order description of tunneling, whereas higher-order reflections would be described by higher-order terms in the SFA.

\section{Quantum reflections and time delay}

What is the origin of this time delay? For longer barriers, $\xi \gg 1$, the tunneling time $\tau$ becomes

$$
\lim _{\xi \gg 1} \tau \approx \frac{2 \chi}{p_{0}^{2}},
$$

vanishing when $\chi \ll 1$. Thus, the time delay is vanishing for $\xi \gg 1$ and $\chi \ll 1$. With this knowledge, we can analyze the exact wave function inside the barrier. The reflection coefficient $C_{1}$ in the given limiting conditions, $\xi \gg 1$ and $\chi \ll 1$,

$$
C_{1} \approx-2 i \chi e^{-2 \xi}
$$

vanishes asymptotically. This suggests an intuitive link between the reflection of wave components and the retardation of the wave packet.

In the box potential case, in contrast to the tunneling problem, there exists, however, a second reflection: the reflection from incidence on the first surface of the barrier, described by the coefficient $R$. Classically, $R=-1$, and there is no time delay at reflection. However, in the quantum case $R+1 \neq 0$, inducing a time delay of the electron wave packet from entry into the barrier. In the limit $\xi \gg 1$ and $\chi \ll 1$, we have $R \approx-(1+2 i \chi)$, i.e.,

$$
|R+1| \approx \chi \ll 1
$$

Analyzing the exact wave function, we can conclude that the time delay vanishes when the reflection from the barrier surface is classical, and when the reflection inside the barrier is negligible.

One can deduce the time delay caused by reflection at the entry by applying the Wigner delay formula, Eq. (1), at $x=0$. For $\xi \gg 1, R \approx-(1+i \chi) /(1-i \chi)$ and

$$
\tau_{\text {entry }}=\left.\frac{-i}{p_{0}} \frac{\partial}{\partial p} \ln \left(\frac{\psi_{\mathrm{I}}(x)}{\left|\psi_{\mathrm{I}}(x)\right|}\right)\right|_{x=0} \approx \frac{\chi}{p_{0}^{2}}=\frac{1}{2} \lim _{\xi \gg 1} \tau .
$$

Thus, one can conclude the time taken for the peak of the electron wave packet to travel through the box potential is caused in equal parts by the reflections of the wave packet on the barrier entry $(x=0)$ and exit $(x=a)$ surfaces.

\section{APPENDIX B: CONFIGURATIONS OF SADDLE POINTS}

The configurations of the saddle points of the argument $\Phi\left(x, t, t^{\prime}\right)$ of the wave-function integral $\psi_{i}=\int_{t_{0}}^{t} d t^{\prime} \exp (i \Phi)$ in the complex $t^{\prime}$ plane, for a large $(x, t)$ parameter range, are presented as a table in Fig. 11. The laser pulse evolves from left to right in this figure and mostly shifts the reference line around which the saddle points are centered, namely, $\operatorname{Re}\left[t^{\prime}\right]=t$. It should be noted, by the definition in Eq. (17), that a singularity is always to be observed at the point $t^{\prime}=t$.

As one moves down the table, configurations of the complex $t^{\prime}$ plane are shown for spatial coordinates inside, neighboring, and outside the tunneling barrier $(x=7,10$, and 14 a.u., respectively). There are marked differences between each observation coordinate but the behavior is reminiscent of the complex plane configuration for the Airy integral, displayed in Fig. 5. Indeed, the two pictures can easily be reconciled by a $\pi / 2-\mathrm{rad}$ clockwise rotation.

Inside the barrier, the saddle points are vertically aligned along the line $\operatorname{Re}\left[t^{\prime}\right]=t$. As one increases the observation coordinate and approaches the tunnel exit, $x \approx 10$, the two saddle points approach each other vertically; as one exits the region neighboring the tunnel exit, $x \gg 10$, the saddles then separate from each other on the horizontal.

As one approaches the peak of the laser field, $t=0$, the distance of closest approach around the tunnel exit shrinks. At the exact peak, this distance is zero; that is, the saddle points merge. This phenomenon is directly comparable to the merging of saddle points for the Airy integral at the classical tunnel exit. Thus, we are able identify a new tunnel exit, $x_{t}$, from the complex plane landscape, a topic discussed in Sec. IV B. 

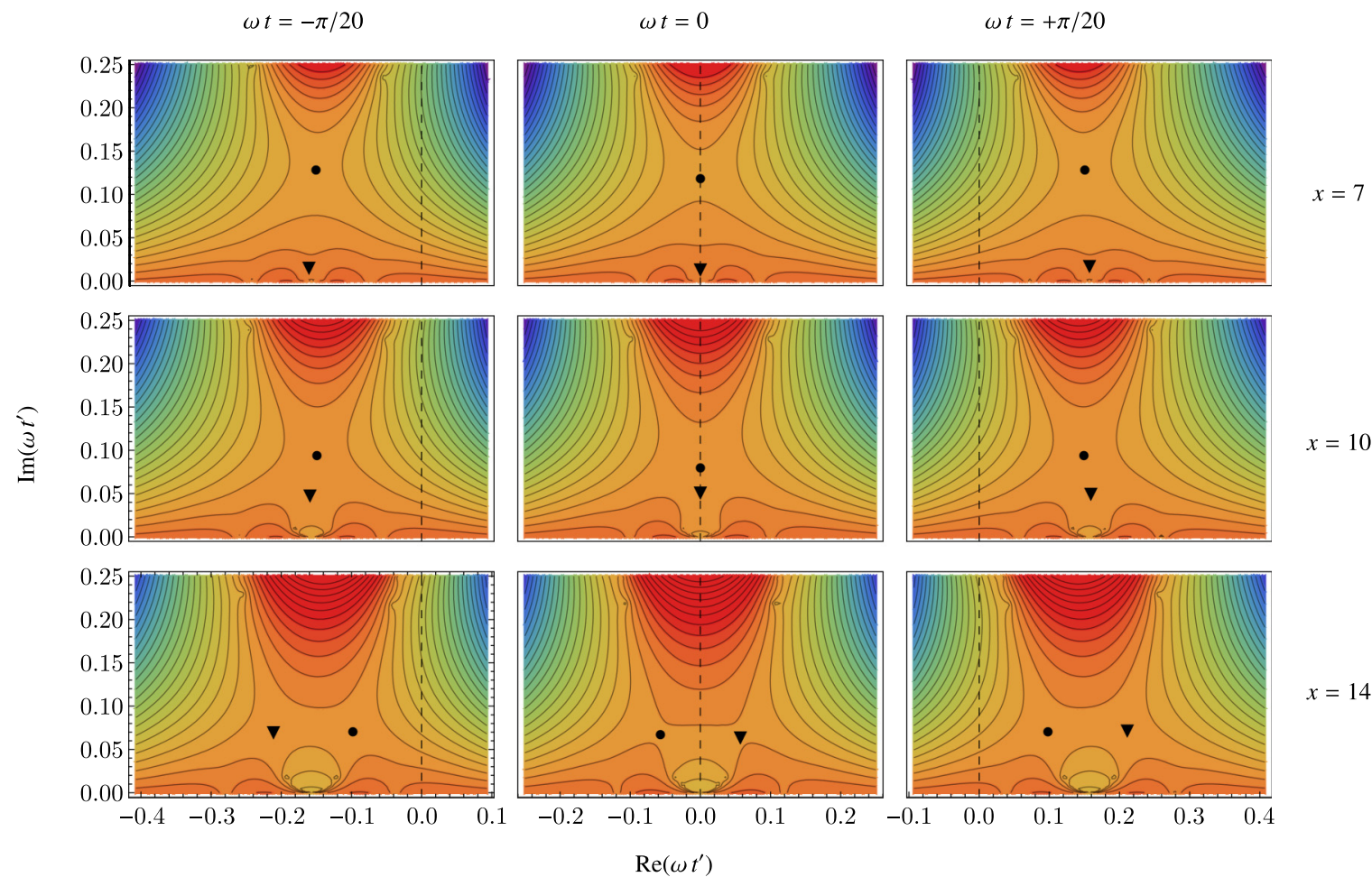

FIG. 11. Configurations of the saddle points of the argument $\Phi\left(x, t, t^{\prime}\right)$ of the wave-function integral $\psi_{i}=\int_{t_{0}}^{t} d t^{\prime} \exp (i \Phi)$ in the complex $t^{\prime}$ plane, for parameter ranges $x=7,10,14$ and $\omega t=-\pi / 20,0,+\pi, 20$. The dashed line corresponds to $t^{\prime}=0$ and each plot is centered around the (scaled) observation time $\omega t$. The scale and color coding are identical to those of Fig. 6 . As $x$ increases the two saddle points approach vertically and, after a closest approach, separate horizontally. For the peak of the pulse, $\omega t=0$, this closest approach is zero and the two saddle points merge at the point $x_{t}$. Otherwise, varying $t$ skews the relative orientation of the saddle points around the line $\operatorname{Re}\left[t^{\prime}\right]=t$.

The identification of the exact value for the coordinate $x_{t}$ to a desired precision is in principle achievable by a binary search or global minimization of the dis- tance function for the saddle points. However, such precision was deemed unnecessary for the purposes of this paper.
[1] L. A. MacColl, Note on the transmission and reflection of wave packets by potential barriers, Phys. Rev. 40, 621 (1932).

[2] A. Peres, Measurement of time by quantum clocks, Am. J. Phys. 48, 552 (1980).

[3] R. Landauer and T. Martin, Barrier interaction time in tunneling, Rev. Mod. Phys. 66, 217 (1994).

[4] E. H. Hauge and J. A. Støvneng, Tunneling times: A critical review, Rev. Mod. Phys. 61, 917 (1989).

[5] Time in Quantum Mechanics, edited by J. G. Muga, I. L. Egusquiza, and R. S. Mayato, Lecture Notes in Physics Vol. 72 (Springer, New York, 2002).

[6] C. A. A. de Carvalho and H. M. Nussenzveig, Time delay, Phys. Rep. 364, 83 (2002).

[7] P. C. W. Davies, Quantum tunneling time, Am. J. Phys. 73, 23 (2005).

[8] H. G. Winful, Tunneling time, the Hartman effect, and superluminality: A proposed resolution of an old paradox, Phys. Rep. 436, 1 (2006).

[9] D. Sokolovski and E. Akhmatskaya, "Superluminal paradox" in wave packet propagation and its quantum mechanical resolution, Ann. Phys. (NY) 339, 307 (2013).
[10] L. Eisenbud, The formal properties of nuclear collisions, Ph.D. thesis, Princeton University, 1948.

[11] E. P. Wigner and L. Eisenbud, Higher angular momenta and long range interaction in resonance reactions, Phys. Rev. 72, 29 (1947).

[12] F. T. Smith, Lifetime matrix in collision theory, Phys. Rev. 118, 349 (1960).

[13] E. P. Wigner, Lower limit for the energy derivative of the scattering phase shift, Phys. Rev. 98, 145 (1955).

[14] A. Czirják, R. Kopold, W. Becker, M. Kleber, and W. Schleich, The Wigner function for tunneling in a uniform static electric field-Dedicated to Marlan O. Scully on the occasion of his 60th birthday, Opt. Commun. 179, 29 (2000).

[15] M. Büttiker and R. Landauer, Traversal Time for Tunneling, Phys. Rev. Lett. 49, 1739 (1982).

[16] E. Pollak and W. H. Miller, New Physical Interpretation for Time in Scattering Theory, Phys. Rev. Lett. 53, 115 (1984).

[17] A. I. Baz', Lifetime of intermediate states, Yad. Fiz. 4 (1966).

[18] V. Rybachenko, Time of penetration of a particle through a potential barrier, Sov. J. Nucl. Phys. 5, 635 (1967). 
[19] M. Büttiker, Larmor precession and the traversal time for tunneling, Phys. Rev. B 27, 6178 (1983).

[20] M. Deutsch and J. E. Golub, Optical Larmor clock: Measurement of the photonic tunneling time, Phys. Rev. A 53, 434 (1996).

[21] R. Ramos, D. Spierings, and I. T. Racicot, Measurement of the time spent by a tunnelling atom within the barrier region, Nature (London) 583, 529 (2020).

[22] H. M. Nussenzveig, Average dwell time and tunneling, Phys. Rev. A 62, 042107 (2000).

[23] D. Sokolovski and L. M. Baskin, Traversal time in quantum scattering, Phys. Rev. A 36, 4604 (1987).

[24] A. M. Steinberg, How Much Time does a Tunneling Particle Spend in the Barrier Region?, Phys. Rev. Lett. 74, 2405 (1995).

[25] N. Yamada, Unified Derivation of Tunneling Times from Decoherence Functionals, Phys. Rev. Lett. 93, 170401 (2004).

[26] A. S. Landsman and U. Keller, Attosecond science and the tunnelling time problem, Phys. Rep. 547, 1 (2015).

[27] L. Maccone and K. Sacha, Quantum Measurements of Time, Phys. Rev. Lett. 124, 110402 (2020).

[28] P. Eckle, M. Smolarski, F. Schlup, J. Biegert, A. Staudte, M. Schöffler, H. G. Muller, R. Dörner, and U. Keller, Attosecond angular streaking, Nat. Phys. 4, 565 (2008).

[29] P. Eckle, A. N. Pfeiffer, C. Cirelli, A. Staudte, R. Dörner, H. G. Muller, M. Büttiker, and U. Keller, Attosecond ionization and tunneling delay time measurements in helium, Science 322, 1525 (2008).

[30] A. N. Pfeiffer, C. Cirelli, M. Smolarski, D. Dimitrovski, M. Abu-Samha, L. B. Madsen, and U. Keller, Attoclock reveals natural coordinates of the laser-induced tunnelling current flow in atoms, Nat. Phys. 8, 76 (2012).

[31] A. S. Landsman, M. Weger, J. Maurer, R. Boge, A. Ludwig, S. Heuser, C. Cirelli, L. Gallmann, and U. Keller, Ultrafast resolution of tunneling delay time, Optica 1, 343 (2014).

[32] U. S. Sainadh, H. Xu, X. Wang, A. Atia-Tul-Noor, W. C. Wallace, N. Douguet, A. Bray, I. Ivanov, K. Bartschat, A. Kheifets, R. T. Sang, and I. V. Litvinyuk, Attosecond angular streaking and tunnelling time in atomic hydrogen, Nature (London) 568, 75 (2019).

[33] N. Camus, E. Yakaboylu, L. Fechner, M. Klaiber, M. Laux, Y. Mi, K. Z. Hatsagortsyan, T. Pfeifer, C. H. Keitel, and R. Moshammer, Experimental Evidence for Wigner's Tunneling Time, Phys. Rev. Lett. 119, 023201 (2017).

[34] N. Teeny, E. Yakaboylu, H. Bauke, and C. H. Keitel, Ionization Time and Exit Momentum in Strong-Field Tunnel Ionization, Phys. Rev. Lett. 116, 063003 (2016).

[35] N. Teeny, C. H. Keitel, and H. Bauke, Virtual-detector approach to tunnel ionization and tunneling times, Phys. Rev. A 94, 022104 (2016).

[36] E. Yakaboylu, M. Klaiber, H. Bauke, K. Z. Hatsagortsyan, and C. H. Keitel, Relativistic features and time delay of laserinduced tunnel ionization, Phys. Rev. A 88, 063421 (2013).

[37] M. Han, P. Ge, Y. Fang, X. Yu, Z. Guo, X. Ma, Y. Deng, Q. Gong, and Y. Liu, Unifying Tunneling Pictures of Strong-Field Ionization with an Improved Attoclock, Phys. Rev. Lett. 123, 073201 (2019).

[38] G. Orlando, C. R. McDonald, N. H. Protik, G. Vampa, and T. Brabec, Tunnelling time, what does it mean?, J. Phys. B 47, 204002 (2014).
[39] G. Orlando, C. R. McDonald, N. H. Protik, and T. Brabec, Identification of the Keldysh time as a lower limit for the tunneling time, Phys. Rev. A 89, 014102 (2014).

[40] M. Lein, Streaking analysis of strong-field ionisation, J. Mod. Opt. 58, 1188 (2011).

[41] A. S. Landsman and U. Keller, Tunnelling time in strong field ionisation, J. Phys. B 47, 204024 (2014).

[42] L. Torlina, F. Morales, J. Kaushal, I. Ivanov, A. Kheifets, A. Zielinski, A. Scrinzi, H. G. Muller, S. Sukiasyan, M. Ivanov, and O. Smirnova, Interpreting attoclock measurements of tunnelling times, Nat. Phys. 11, 503 (2015).

[43] H. Ni, U. Saalmann, and J.-M. Rost, Tunneling exit characteristics from classical backpropagation of an ionized electron wave packet, Phys. Rev. A 97, 013426 (2018).

[44] H. Ni, N. Eicke, C. Ruiz, J. Cai, F. Oppermann, N. I. ShvetsovShilovski, and L.-W. Pi, Tunneling criteria and a nonadiabatic term for strong-field ionization, Phys. Rev. A 98, 013411 (2018).

[45] H. Ni, U. Saalmann, and J.-M. Rost, Tunneling Ionization Time Resolved by Backpropagation, Phys. Rev. Lett. 117, 023002 (2016).

[46] C. Hofmann, A. Bray, W. Koch, H. Ni, and N. I. ShvetsovShilovski, Quantum battles in attoscience: Tunnelling, Eur. Phys. J. D 75, 208 (2021).

[47] A. M. Zheltikov, Keldysh parameter, photoionization adiabaticity, and the tunneling time, Phys. Rev. A 94, 043412 (2016).

[48] T. Zimmermann, S. Mishra, B. R. Doran, D. F. Gordon, and A. S. Landsman, Tunneling Time and Weak Measurement in Strong Field Ionization, Phys. Rev. Lett. 116, 233603 (2016).

[49] J. Liu, Y. Fu, W. Chen, Z. Lü, J. Zhao, J. Yuan, and Z. Zhao, Offset angles of photocurrents generated in few-cycle circularly polarized laser fields, J. Phys. B 50, 55602 (2017).

[50] Y. Song, Y. Yang, F. Guo, and S. Li, Revisiting the timedependent ionization process through the Bohmian-mechanics method, J. Phys. B 50, 95003 (2017).

[51] M. Yuan, P. Xin, T. Chu, and H. Liu, Exploring tunneling time by instantaneous ionization rate in strong-field ionization, Opt. Express 25, 23493 (2017).

[52] M. Klaiber, K. Z. Hatsagortsyan, and C. H. Keitel, Underthe-Tunneling-Barrier Recollisions in Strong-Field Ionization, Phys. Rev. Lett. 120, 013201 (2018).

[53] N. Eicke and M. Lein, Trajectory-free ionization times in strong-field ionization, Phys. Rev. A 97, 031402(R) (2018).

[54] N. Douguet and K. Bartschat, Dynamics of tunneling ionization using bohmian mechanics, Phys. Rev. A 97, 013402 (2018).

[55] A. W. Bray, S. Eckart, and A. S. Kheifets, Keldysh-Rutherford Model for the Attoclock, Phys. Rev. Lett. 121, 123201 (2018).

[56] B. Baytaş, M. Bojowald, and S. Crowe, Canonical tunneling time in ionization experiments, Phys. Rev. A 98, 063417 (2018).

[57] X. Ren, Y. Wu, L. Wang, and Y. Zheng, Entangled trajectories during ionization of an $\mathrm{H}$ atom driven by n-cycle laser pulse, Phys. Lett. A 382, 2662 (2018).

[58] J. Tan, Y. Zhou, M. He, Y. Chen, Q. Ke, J. Liang, X. Zhu, M. Li, and P. Lu, Determination of the Ionization Time Using Attosecond Photoelectron Interferometry, Phys. Rev. Lett. 121, 253203 (2018).

[59] D. Sokolovski and E. Akhmatskaya, No time at the end of the tunnel, Commun. Phys. 1, 47 (2018). 
[60] W. Quan, V. V. Serov, M. Z. Wei, M. Z. Zhao, Y. Zhou, Y. L. Wang, X. Y. Lai, A. S. Kheifets, and X. J. Liu, Attosecond Molecular Angular Streaking with All-Ionic Fragments Detection, Phys. Rev. Lett. 123, 223204 (2019).

[61] N. Douguet and K. Bartschat, Attoclock setup with negative ions: A possibility for experimental validation, Phys. Rev. A 99, 023417 (2019).

[62] C. Hofmann, A. S. Landsman, and U. Keller, Attoclock revisited on electron tunnelling time, J. Mod. Opt. 66, 1052 (2019).

[63] V. V. Serov, A. W. Bray, and A. S. Kheifets, Numerical attoclock on atomic and molecular hydrogen, Phys. Rev. A 99, 063428 (2019).

[64] R. Wang, Q. Zhang, D. Li, S. Xu, P. Cao, Y. Zhou, W. Cao, and P. Lu, Identification of tunneling and multiphoton ionization in intermediate Keldysh parameter regime, Opt. Express 27, 6471 (2019).

[65] M. Yuan, Direct probing of tunneling time in strongfield ionization processes by time-dependent wave packets, Opt. Express 27, 6502 (2019).

[66] A. S. Kheifets, The attoclock and the tunneling time debate, J. Phys. B 53, 72001 (2020).

[67] S. Yusofsani and M. Kolesik, Quantum tunneling time: Insights from an exactly solvable model, Phys. Rev. A 101, 052121 (2020).

[68] B. Feuerstein and U. Thumm, On the computation of momentum distributions within wavepacket propagation calculations, J. Phys. B 36, 707 (2003).

[69] X. Wang, J. Tian, and J. H. Eberly, Extended Virtual Detector Theory for Strong-Field Atomic Ionization, Phys. Rev. Lett. 110, 243001 (2013).

[70] K. Liu, H. Ni, K. Renziehausen, J.-M. Rost, and I. Barth, Deformation of Atomic $p_{ \pm}$Orbitals in Strong Elliptically Polarized
Laser Fields: Ionization Time Drifts and Spatial Photoelectron Separation, Phys. Rev. Lett. 121, 203201 (2018).

[71] H. Ni, S. Brennecke, X. Gao, P.-L. He, S. Donsa, I. Březinová, F. He, J. Wu, M. Lein, X.-M. Tong, and J. Burgdörfer, Theory of Subcycle Linear Momentum Transfer in Strong-Field Tunneling Ionization, Phys. Rev. Lett. 125, 073202 (2020).

[72] D. Bohm, Quantum Theory (Prentice-Hall, Englewood Cliffs, NJ, 1951).

[73] L. V. Keldysh, Ionization in the field of a strong electromagnetic wave, Zh. Eksp. Teor. Fiz. 47, 1945 (1964).

[74] M. Klaiber, J. Daněk, E. Yakaboylu, K. Z. Hatsagortsyan, and C. H. Keitel, Strong-field ionization via a high-order Coulombcorrected strong-field approximation, Phys. Rev. A 95, 023403 (2017).

[75] A more precise definition for the tunnel exit can be given by the SFA, namely, $x_{e}^{\mathrm{SFA}}=\int_{t_{s}}^{\operatorname{Re}\left\{\mathrm{t}_{\mathrm{s}}\right\}}\left[A\left(t^{\prime}\right)-A(0)\right] d t^{\prime} \approx(1-$ $\left.\gamma^{2} / 4\right) I_{p} / E_{0}=0.995 I_{p} / E_{0}$, where $t_{s}$ is the temporal saddle point of the SFA integral. The minuscule discrepancy between the classical tunnel exit, $x_{e}$, and its SFA correction for the parameters in this paper $(\gamma=\sqrt{2} / 10)$ leads us to use the simpler of the two definitions.

[76] W. Becker, F. Grasbon, R. Kopold, D. B. Milošević, G. G. Paulus, and H. Walther, Above-threshold ionization: From classical features to quantum effects, Adv. At. Mol. Opt. Phys. 48, 35 (2002).

[77] D. M. Wolkow, Über eine Klasse von Lösungen der Diracschen Gleichung, Z. Phys. 94, 250 (1935).

[78] C. M. Bender and S. A. Orszag, Advanced Mathematical Methods for Scientists and Engineers (McGraw-Hill, New York, 1978).

[79] C. Chester, B. Friedman, and F. Ursell, An extension of the method of steepest descents, Math. Proc. Cambridge Philos. Soc. 53, 599 (1957). 